\title{
Neoadjuvant chemotherapy in bladder cancer: not a matter on how much but on who it is effective
}

\author{
Giovanni Motterle, R. Jeffrey Karnes \\ Department of Urology, Mayo Clinic, Rochester, MN, USA \\ Correspondence to: R. Jeffrey Karnes, MD. Department of Urology, Mayo Clinic, 200 1st St SW, Rochester, MN 55905, USA. Email: Karnes.R@mayo.edu. \\ Provenance: This is an invited article commissioned by Section Editor Xiao Li (Department of Urology, Jiangsu Cancer Hospital \& Jiangsu Institute \\ of Cancer Research \& Nanjing Medical University Affiliated Cancer Hospital, Nanjing, China). \\ Comment on: Waingankar N, Jia R, Marqueen KE, et al. The impact of pathologic response to neoadjuvant chemotherapy on conditional survival \\ among patients with muscle-invasive bladder cancer. Urol Oncol 2019;37:572.e21-572.e28.
}

Submitted Aug 27, 2019. Accepted for publication Sep 22, 2019.

doi: $10.21037 /$ tau.2019.09.39

View this article at: http://dx.doi.org/10.21037/tau.2019.09.39

In this study published in Urologic Oncology, Waingankar and the group form Mount Sinai (1) perform an analysis on the impact of pathological complete response to neoadjuvant chemotherapy (NAC) in muscle-invasive bladder cancer (MIBC) on conditional surviving, meaning the updated probability of surviving after having survived each additional year after the treatment.

This study directly follows the first findings of Sun et al. (2) and Ploussard et al. (3) and the key message remains the same: mortality risk changes over time and mortality decreases with increasing survivorship.

The originality of this study comes from its aim, namely determining the impact of pathological response to NAC. In order to achieve this, the authors used the National Cancer Database to obtain their analytic cohort of 1,553 patients; this strategy permitted to include in the analysis a great amount of demographic and socioeconomic factors but lacked of follow-up information, eventually allowing to perform analyses based only on overall survival, whereas the previous aforementioned studies included cancer-specific survival. The latter seems clearly more indicated in this setting, even if comorbidities were comparable between responders and non-responders.

This study reports a rate of pathological downstaging of approximately $20 \%$, defined as $<\mathrm{pT} 1$ disease with negative nodes. The stringent criteria used for evaluating the main outcome is somehow contrasting with the more wide inclusion of patients undergoing any multi-agent NAC, apparently independently from the use of cisplatin.

Apart from these considerations, a strong statistical analysis showed what expected: conditional survival probabilities improve with time both for responders and non-responders. More interestingly the effect of pathological complete response is sustained over time, in particular in the first three years.

The take home message from this study probably will not affect clinical practice, since it is a common perception that patients who respond to NAC tend to "do well" also over time. Now we can prove this "perception" with numbers. What remains to be defined, and should guide future research, is why some patients tend to benefit from NAC and others do not. Changing the criteria for defining response seems to affect only relatively its implication on survival (4) and, in our opinion, we should not forget that radical cystectomy maintains its role in eradicating the primary disease provided that negative margins are achieved, while NAC controls potential micro-metastatic disease; thus failure to achieve pT0 disease should not be regarded as a complete defeat.

In the last years, much effort was put into trying to define a strategy to predict the response to cisplatin-based NAC and definitive answers are yet to be found. The same group from Mount Sinai indeed proposed an interesting review of the progress in understanding bladder cancer biology (5) highlighting the heterogeneity of the disease, since the first hypotheses of different pathways involved in the development of NMIBC and MIBC, to genetic mutations, up to modern clustering of tumors subtypes based on RNA expression. The latest comprehensive molecular characterizations of bladder cancer $(6,7)$ might 
not be the easiest readings and ready-applicable for everyday clinical practice, but gives us the idea that bladder cancer is one of the cancers carrying the highest mutational load but some of the mutations are potentially amenable to therapeutic targeting. In particular five different subtypes of bladder cancer were defined and some of them seemed to be associated with lower response to cisplatin based chemotherapy.

In light of these considerations, pathological response could be interpreted as a surrogate sign of a particular subtype of tumors for which current regimens of chemotherapy are the most suitable treatment in addition to cystectomy; its positive impact on conditional survival might provide an additional proof of it.

Are we then ready to change our follow-up strategies for patients achieving pathological complete response at the time of radical cystectomy after NAC? Not yet; at least until we could offer a "tailored" and targeted alternative to cisplatin in the neoadjuvant setting or in the adjuvant second-line regimens.

As already pointed out by Gandaglia and Sun (8) the concept of conditional survival should however raise the consciousness on the importance of timing and of multimodality treatment in such a complex disease. NAC use is increased in the least years $(9,10)$ but there is still room for improvement. Results from the study of Waingankar et al. should then be regarded as an additional proof of its benefits in some patients but also as an additional call for effort in defining who these patients are.

\section{Acknowledgments}

None.

\section{Footnote}

Conflicts of Interest: The authors have no conflicts of interest to declare.

Ethical Statement: The authors are accountable for all aspects of the work in ensuring that questions related to the accuracy or integrity of any part of the work are appropriately investigated and resolved.

\section{References}

1. Waingankar N, Jia R, Marqueen KE, et al. The impact of pathologic response to neoadjuvant chemotherapy on conditional survival among patients with muscle-invasive bladder cancer. Urol Oncol 2019;37:572.e21-572.e28.

2. Sun M, Abdollah F, Bianchi M, et al. Conditional survival of patients with urothelial carcinoma of the urinary bladder treated with radical cystectomy. Eur J Cancer 2012;48:1503-11.

3. Ploussard G, Shariat SF, Dragomir A, et al. Conditional survival after radical cystectomy for bladder cancer: evidence for a patient changing risk profile over time. Eur Urol 2014;66:361-70.

4. Rosenblatt R, Sherif A, Rintala E, et al. Pathologic downstaging is a surrogate marker for efficacy and increased survival following neoadjuvant chemotherapy and radical cystectomy for muscle-invasive urothelial bladder cancer. Eur Urol 2012;61:1229-38.

5. Audenet F, Attalla K, Sfakianos JP. The evolution of bladder cancer genomics: What have we learned and how can we use it?. Urol Oncol 2018;36:313-20.

6. Cancer Genome Atlas Research Network. Comprehensive molecular characterization of urothelial bladder carcinoma. Nature 2014;507:315-22.

7. Robertson AG, Kim J, Al-Ahmadie H, et al. Comprehensive Molecular Characterization of MuscleInvasive Bladder Cancer. Cell 2017;171:540-56.e25.

8. Gandaglia G, Sun M. Bladder cancer: Conditional survival after radical cystectomy. Nat Rev Urol 2014;11:8-9.

9. Reardon ZD, Patel SG, Zaid HB, et al. Trends in the use of perioperative chemotherapy for localized and locally advanced muscle-invasive bladder cancer: a sign of changing tides. Eur Urol 2015;67:165-70.

10. Hermans TJN, Fransen van de Putte EE, Horenblas $S$, et al. Perioperative treatment and radical cystectomy for bladder cancer--a population based trend analysis of 10,338 patients in the Netherlands. Eur J Cancer 2016;54:18-26.
Cite this article as: Motterle G, Karnes RJ. Neoadjuvant chemotherapy in bladder cancer: not a matter on how much but on who it is effective. Transl Androl Urol 2019;8(Suppl 5):S480-S481. doi: 10.21037/tau.2019.09.39 